\title{
ASSESSMENT OF DRUG USE FOR INPATIENTS WHO WERE PRESCRIBED CEFTAZIDIME: A RETROSPECTIVE CROSS-SECTIONAL STUDY IN A DISTRICT-LEVEL HOSPITAL
}

\section{ABSTRACT}

In health facilities, there is a lot of public concern about the irrational use of medications, especially antibiotic misuse. This retrospective cross-sectional study was conducted to assess the utilization of medicines for inpatients using ceftazidime in treatment in a district-level hospital in Vietnam, using 396 medical records. The results showed that there were approximately 6.7 kinds of drugs prescribed for each inpatient, including roughly 1.23 antibiotics. On average, 5.69 days of hospitalization cost an inpatient about 74.31USD, including more than 20USD for medicines (9.22USD for antibiotics) and 54.27USD for other expenses. There were $19.70 \%$ of medical records in which found at least one drug-drug interaction. Cardiovascular medicines, corticoids, fluoroquinolones and proton-pump inhibitors were medicine groups involving many found drug-drug interactions. For ceftazidime, indications of this antibiotic were inappropriate for nearly half of inpatients. For 199 inpatients whose indications' ceftazidime were appropriate, the route of ceftazidime administration was appropriate but its dose per day and dose duration were inappropriate. More importantly, ceftazidime was used with a dosage lower than the recommended dosage (98.99\%). In conclusion, there were several problems involving drug use that should be addressed, including curbing major drug-drug interactions and the irrational use of ceftazidime, a watchgroup, broad-spectrum antibiotic.

Keywords: Drug use, antibiotic, ceftazidime, inpatient, district-level hospital.

\footnotetext{
* Hanoi University of Pharmacy
}

Responsible person: Nguyen Thi Thanh Huong Email: huongnguyenthanh@ hup.edu.vn

Date of receipt: 03/5/2021

Date of scientific judgment: 19/6/2021

Reviewed date: 01/8/2021

\author{
Nguyen Thi Thanh Huong*, Dinh Xuan Dai*
}

\section{BACKGROUND}

At present, an intractable problem occurring in many countries is the irrational use of medicines (especially antibiotics) that can engender severe consequences like causing deaths or contributing to the alarming increase of antibiotic resistance. The World Health Organization (WHO) announced that there was a shortage of new antibiotics under development to struggle against the growing menace of antimicrobial resistance. In 2017, the $21^{\text {st }}$ meeting of the WHO Expert Committee took place in Switzerland to review and update model lists of essential medicines and propose a new categorization of antibacterials into three groups (access, watch and reserve) [8].

Ceftazidime is a third-generation, broadspectrum cephalosporin, being used for treating complicated infections (like pneumonia, urinary infection, sepsis). A wide range of gram-negative bacteria is vulnerable to this antibiotic, including strains resistant to aminoglycosides like Pseudomonas. The significant feature to distinguish ceftazidime from other cephalosporins is its activity against Pseudomonas aeruginosa, a harmful bacterium usually giving rise to nosocomial infections [3], [6]. Thanks to these salient characteristics, this watch-group antibiotic has become one of the popularly used cephalosporins in health facilities. 
Drug use evaluation should be done frequently in hospitals to ensure the rational use of drugs. The frequent assessment of antibiotic use can assist health facilities to enhance treatment efficiency, lower treatment expenditure and the length of hospitalization, hinder unwanted adverse drug reactions and antibiotic resistance. In hospitals, there is a plethora of conducted researches involving the use of other cephalosporins while data on ceftazidime are negligible, especially in Vietnam. This study was conducted to assess the reality of using ceftazidime and other medications for inpatients in a district-level hospital, thereby proposing several practical solutions, making contributions to guaranteeing rational drug use and putting a curb on the increase of antibiotic resistance.

\section{METHODOLOGY}

This study was a retrospective crosssectional study conducted in Vinhthuan General Hospital located in Kiengiang, a western province in the Mekong River Delta area of Vietnam. Prior to the process of data collection, researchers obtained approval to conduct this study from Hanoi University of Pharmacy and permission from the director of Vinhthuan General Hospital to make use of their database. Data acquired were also kept anonymized (The inpatients' names and their addresses were not collected or used).

From 1st January 2018 to 31st December 2018, 2,075 patients were hospitalized and used ceftazidime. Their medical records were enumerated in order by date of hospitalization. In this study, 406 medical records were chosen using systematic random sampling with $\mathrm{k}=5.11$. However, after being checked, ten medical records were excluded by virtue of the lack of necessary information for data analysis.

Data on prescribed medicines were entered and analyzed using Microsoft Excel 2020 and $\mathrm{R}$ version 4.0.3. Continuous data were reported as mean and standard deviation (SD) while categorical data were reported as absolute values (n) and proportions (\%). Analysis of ceftazidime use was compared to the guideline on antibiotic use published by the Vietnam Ministry of Health in 2015 [7]. Drug-drug interactions (DDIs) were checked using Micromedex online. In this study, contraindicated, major and moderate DDIs were included while minor DDIs were excluded. As per Micromedex: Contraindicated DDIs (The drugs are contraindicated for concurrent use); Major DDIs (The interactions may be lifethreatening and/or require medical intervention to minimize or prevent serious adverse effects) and Moderate DDIs (The interaction may result in exacerbation of the patient's condition and/or require an alteration in therapy).

\section{RESULTS}

Nearly half of the inpatients were senior adults aged 60 and older. The percentage of male patients was higher than that of female patients, by roughly $8 \%$. Most of the patients came from the Department of Pediatrics and Internal Medicine. On average, a patient contracted two kinds of ailments. Infections that a multitude of patients contracted included respiratory and gastrointestinal infections (Table 1). 
No1\&2/2021 VIETNAM MEDICAL JOURNAL

\begin{tabular}{|l|l|r|}
\hline \multicolumn{3}{|c|}{ Table 1. Characteristics of 396 inpatients } \\
\hline \multirow{4}{*}{ Indicators } & \multicolumn{2}{|c|}{ Value n (\%) } \\
\cline { 2 - 3 } & Mean \pm SD & $43.06 \pm 33.73$ \\
\cline { 2 - 3 } & Child (0-12 years) & $151(38,13 \%)$ \\
\cline { 2 - 3 } & Adolescence (13-18 years) & $6(1,52 \%)$ \\
\cline { 2 - 3 } & Adult (19-59 years) & $48(12,12 \%)$ \\
\cline { 2 - 3 } & Senior adult (60 years and above) & $191(48,23 \%)$ \\
\hline \multirow{5}{*}{ Gender } & Male & $214(54,04 \%)$ \\
\cline { 2 - 3 } & Female & $182(45,96 \%)$ \\
\hline \multirow{2}{*}{ Height } & Mean \pm SD & $131.02 \pm 37.92$ \\
\hline \multirow{2}{*}{ Deight } & Mean \pm SD & $37.74 \pm 21.81$ \\
\hline \multirow{3}{*}{ Department } & Mean \pm SD & $5.69 \pm 2.58$ \\
\hline \multirow{5}{*}{ Common diseases } & Pediatrics & $154(38,89 \%)$ \\
\cline { 2 - 3 } & Surgery & $11(2,78 \%)$ \\
\cline { 2 - 3 } & Internal Medicine & $187(47,22 \%)$ \\
\cline { 2 - 3 } & Infectious Diseases & $44(11,11 \%)$ \\
\cline { 2 - 3 } & Respiratory infection & $274(69,19 \%)$ \\
\cline { 2 - 3 } & Gastrointestinal infection & $142(35,86 \%)$ \\
\cline { 2 - 3 } & Infectious diarrhea & $59(14,90 \%)$ \\
\cline { 2 - 3 } & Chronic obstructive pulmonary disease & $42(10,61 \%)$ \\
\hline
\end{tabular}

On average, an inpatient took 6.7 20.04USD for medicines (Exchange-rate 1 medicines per course of treatment. There USD $=23,195$ vietnam dong). The cost of were 316, 72, 7 and 1 patients who used one, antibiotics (mainly sprung from ceftazidime) two, three and five antibiotics during their constituted roughly $11.61 \%$ of the cost of one whole treatment course, respectively. The treatment course and more than half of the average expenditure for one treatment course expenditure of used medicines (Table 2). was approximately 74.31USD, including

Table 2. Drug-use indicators in Vinhthuan General Hospital

\begin{tabular}{|l|r|}
\hline \multicolumn{1}{|c|}{ Indicator } & Value (mean \pm SD) \\
\hline Average number of drugs per treatment course & $6.70 \pm 3.01$ \\
\hline Average number of antibiotics per treatment course & $1.23 \pm 0.49$ \\
\hline Average cost for a treatment course (vietnam dong) & $1,723,677 \pm 960,626$ \\
\hline Average cost of drugs per treatment course (vietnam dong) & $464,943 \pm 419,120$ \\
\hline Average cost of antibiotics per treatment course (vietnam dong) & $213,942 \pm 219,141$ \\
\hline Cost of antibiotics per cost of one treatment course (\%) & $11.61 \pm 4.33$ \\
\hline Cost of ceftazidime per cost of one treatment course (\%) & $10.75 \pm 3.16$ \\
\hline Cost of antibiotics per cost of drugs used (\%) & $51.39 \pm 17.23$ \\
\hline Cost of ceftazidime per cost of drugs used (\%) & $48.95 \pm 18.22$ \\
\hline
\end{tabular}

There were 78 medical records in which diseases were the group that usually gave rise found at least one DDI. There were 189 DDIs to DDIs. For instance, the number of DDIs found, including one contraindicated DDI, caused by clopidogrel, spironolactone, 124 major DDIs and 64 moderate DDIs. captopril, furosemide and digoxin were 43, Medicines used to treat cardiovascular 38,34, 22 and 22, respectively (Table 3). 


\section{VIETNAM MEDICAL JOURNAL № $1 \& 2 / 2021$}

Table 3. Drug-drug interactions in 396 medical records

\begin{tabular}{|l|r|}
\hline \multicolumn{1}{|c|}{ Indicator } & \multicolumn{1}{|c|}{ Value } \\
\hline Percentage of medical records with at least one DDI & $19.70 \%$ \\
\hline Average number of DDIs per medical record & 0.47 \\
\hline The number of DDIs involving antibiotics & 42 \\
\hline Contraindicated DDIs (number of cases) & Ceftriaxone + Ringer lactat (1) \\
\hline Major DDIs (number of cases) & Clopidogrel + PPIs* (19) \\
Total: 124 & Corticoid + Fluoroquinolon (15) \\
\cline { 2 - 2 } & Spironolacton + Potassium (15) \\
\cline { 2 - 3 } & Captopril + Potassium (10) \\
\cline { 2 - 3 } Moderate DDIs (number of cases) & Amlodipin + Clopidogrel (9) \\
\cline { 2 - 3 } Total: 64 & Others (56) \\
\cline { 2 - 3 } & Captopril + Furosemid (14) \\
\cline { 2 - 3 } & Clopidogrel + Rosuvastatin (10) \\
\cline { 2 - 3 } & Salbutamol + Spironolacton (10) \\
\cline { 2 - 3 } & Others (30) \\
\cline { 2 - 3 }
\end{tabular}

*PPIs: Proton-pump inhibitors

In 2015, the Vietnam Ministry of Health inappropriate. More importantly, ceftazidime $(\mathrm{VMoH})$ published an antibiotic-use was used with a dosage lower than the guideline to contribute to guaranteeing the recommended dosage (98.99\%). rational use and prescription of antibiotics for Furthermore, ceftazidime was mainly health facilities. However, in this hospital, combined with aminoglycosides and there were only roughly half of inpatients quinolones. The former was mostly used to whose indications of ceftazidime were treat lung inflammation and bronchitis while suitable to this guideline. For these 199 the latter was predominantly employed for inpatients, the route of ceftazidime bronchial asthma, pneumonia and COPD administration was appropriate while its dose (Table 4). per day and the time between doses were

Table 4. Analyzing the use of ceftazidime based on the guideline on antibiotic use published by Vietnam Ministry of Health

\begin{tabular}{|c|c|c|}
\hline Indicator & \multicolumn{2}{|c|}{ Value n (\%) } \\
\hline $\begin{array}{l}\text { The number of cases in which ceftazidime was the } \\
\text { first chosen antibiotic }\end{array}$ & & $358(90.40 \%)$ \\
\hline \multirow{6}{*}{$\begin{array}{l}\text { Combination of ceftazidime and other antibiotics } \\
\text { ( } n \text { umber of cases) } \\
\text { ( } n=39 \text { cases) }\end{array}$} & \multicolumn{2}{|c|}{ Ceftazidime + Gentamycin (19) } \\
\hline & \multicolumn{2}{|c|}{ Ceftazidime + Levofloxacin (10) } \\
\hline & \multicolumn{2}{|c|}{ Ceftazidime + Ciprofloxacin (3) } \\
\hline & \multicolumn{2}{|c|}{ Ceftazidime + Moxifloxacin (3) } \\
\hline & \multicolumn{2}{|c|}{ Ceftazidime + Azithromycin (3) } \\
\hline & \multicolumn{2}{|c|}{ Ceftazidime + Amikacin (1) } \\
\hline \multirow[t]{2}{*}{ Indications } & Inappropriate & $197(49.75 \%)$ \\
\hline & Appropriate & $199(50.25 \%)$ \\
\hline Routes of administration & Appropriate & $199(100 \%)$ \\
\hline \multirow[t]{2}{*}{ Dose per day } & Appropriate & $2(1.01 \%)$ \\
\hline & Inappropriate & $197(98.99 \%)$ \\
\hline \multirow[t]{2}{*}{ Single dose } & Appropriate & $46(23.12 \%)$ \\
\hline & Inappropriate & $153(76.88 \%)$ \\
\hline The time between doses & Inappropriate & $199(100 \%)$ \\
\hline
\end{tabular}




\section{DISCUSSION}

In common, inpatients were individuals contracting severe diseases. Most of the patients in this study were children (38.13\%) and senior citizens $(48.23 \%)$. In the light of the inadequate development in children and the weakened immune system in the elderly, these two patient groups are likely to contract diseases (especially infections) more frequently than other groups. In addition, the latter group usually simultaneously contract many kinds of diseases, vulnerable to various types of infectious diseases such as pneumonia, influenza, urinary tract infection. On average, each inpatient in Vinhthuan General Hospital contracted two kinds of diseases, including an infection (mainly respiratory and gastrointestinal infections) in combination with another disease (like hypertension, diabetes mellitus type 2, hypokalemia).

In one treatment course, an inpatient made use of about 6.7 kinds of medicines, including roughly 1.23 antibiotics. These results were quite similar to those in Eritrea, a country in East Africa: 1.29 antibiotics prescribed per hospitalization and $77 \%$ of patients using one antibiotic [1]. On average, for about 5.69 days of hospitalization, an inpatient had to pay more than 20USD for medications (9.22USD for antibiotics) and 54.27USD for other expenses (mainly costs of sickbed and testings). More importantly, according to the data of General Statistics Office of Vietnam, the monthly average income per capita of people living in the Mekong River Delta area was only about 154.56USD [4]. Seemingly, if hospitalized for the whole month, inhabitants would not be affordable for the total expenditure. It is noteworthy that more than $86 \%$ of inpatients were children who were unable to earn a living by themselves and senior citizens whose incomes were usually negligible.

For DDIs, there were 189 DDIs found in 78 medical records. There were two medical records in which nine DDIs were found, including seven major DDIs. Almost DDIs appeared in patients contracting cardiovascular and gastrointestinal diseases. Quinolones were the antibiotic group that gave rise to DDIs the most. Albeit the low percentage of DDIs in medical records (19.7\%), doctors working in Vinhthuan General Hospital should expand their horizons about DDIs for themselves. This hospital also should promulgate relevant documents, disseminate them to all medical personnel and put a curb on the combination of medicines involving prevalent DDIs.

In Laos, doctors found difficulties in prescribing correct antibiotics (72.5\%). Nearly $60 \%$ of them said that they did not have enough sources of information on antibiotics. In common, doctors obtained information about antibacterial medicines from national guidelines $(86.5 \%)$, peers (85.1\%), pharmaceutical companies $(76.9 \%)$ and the internet $(73.9 \%)$ [5]. For medical personnel residing in rural areas of Vietnam, there was a shortage of competence in using foreign languages. This situation engendered numerous difficulties and hindered their access to medical guidelines and documents. Accordingly, in 2015, with the assistance of many erudite doctors and professors, $\mathrm{VMoH}$ published a guideline on antibiotic use, thereby aiding medical staff in choosing appropriate antibiotics for treating common infections and contributing to curbing the increase of antibiotic resistance.

The results from the comparison of the VMoH's guideline and data from 396 
medical records showed that there were no cases in which ceftazidime was suitably used. For 197 cases in which indications' ceftazidime were inappropriate, this watchgroup antibiotic was predominantly prescribed for children with diarrhea and pneumonia. Ceftazidime can be used for children in pneumonia treatment and many kinds of research were conducted [3], [6]. However, in this guideline, ceftazidime was not recommended to be used for children's pneumonia treatment. VMoH should deeply consider to add this indication of ceftazidime (treating pneumonia for children) to the next version of this guideline. For 199 cases in which indications' ceftazidime were appropriate, there were only 2 cases in which dosage's ceftazidime was suitable to the VMoH's guideline. In other cases, ceftazidime was utilized with a dosage lower than recommendations. Ceftazidime's dosage duration was also inappropriate (100\%) because most of the inpatients were injected with ceftazidime at 8 a.m and 4 p.m.

In rural regions of Vietnam, a multitude of inhabitants are living on the breadline. In health facilities, rarely are antibiograms done. Hence, antibiotics are usually chosen based on doctors' experience. All these conditions can pave the way for antibiotic resistance. Up to now, numerous bacterial strains resisting watch- and reserve-group antibiotics appeared (like colistin-resistant Klebsiella pneumoniae, 3rd-cephalosporinresistant Escherichia coli). Internationally, antibiotic resistance is now a thorny problem. Various kinds of infections became difficult to be treated. In developing countries like Vietnam, rationales behind this problem include poor quality control of available antibiotics; clinical misuse and overuse in hospitals, the community and agriculture; the lack of the surveillance of resistance development and the easy purchase of antibiotics in drugstores by virtue of the lack of retail regulations [2]. As a consequence, the $\mathrm{VMoH}$ and health facilities should frequently organize conferences and workshops to introduce new guidelines and circulars to medical personnel. Furthermore, doctors and pharmacists should broaden their horizons about medicines, especially antibiotics since they play an essential role in widely disseminating the proper use of antibacterial agents to the community.

\section{CONCLUSION}

There were about 6.7 kinds of drugs prescribed for each inpatient, including roughly 1.23 antibiotics. On average, 5.69 days of hospitalization cost an inpatient about 74.31USD, including more than 20USD for medicines (9.22USD for antibiotics) and 54.27USD for other expenses. There were $19.70 \%$ of medical records in which found at least one DDI. Cardiovascular medicines, corticoids, fluoroquinolones and proton-pump inhibitors were medicine groups involving many found DDIs. For ceftazidime, its indications were inappropriate for nearly half of inpatients. For 199 inpatients whose indications' ceftazidime were appropriate, the route of ceftazidime administration was appropriate but its dose per day and dose duration were inappropriate. More importantly, ceftazidime was used with a dosage lower than the recommended dosage $(98.99 \%)$. In conclusion, there were several problems of drug use that should be addressed, including curbing major drug-drug interactions and the irrational use of ceftazidime, a watch-group, broad-spectrum antibiotic. 
No $1 \& 2 / 2021$ VIETNAM MEDICAL JOURNAL

\section{FUNDING}

This research received no specific grant from any funding agencies in the public, commercial or not-for-profit sectors.

\section{CONFLICT OF INTEREST}

The authors declare that there is no conflict of interest.

\section{CONTRIBUTORS}

Huong TTN: Conceptualization, Funding Acquisition, Investigation, Methodology, Project Administration, Resources, Supervision, Writing - Review \& Editing. Dai XD: Conceptualization, Data curation, Formal Analysis, Investigation, Methodology, Software, Validation, Visualization, Writing - Original Draft Preparation, Writing - Review \& Editing.

\section{REFERENCES}

1. Amaha ND, Berhe YH, Kaushik A. Assessment of inpatient antibiotic use in Halibet National Referral Hospital using WHO indicators: a retrospective study. $B M C$ Res Notes. 2018;11(1):904. doi:10.1186/s13104-018-4000-7

2. Chokshi A, Sifri Z, Cennimo D, et al. Global Contributors to Antibiotic Resistance.
Journal of global infectious diseases.

2019;11(1): 36-42. doi:10.4103/jgid.jgid_110_18.

3. Drugbank. Ceftazidime. 2020 Dec 02 [Cited 2020 Dec 03]. Available from: https://go.drugbank.com/drugs/DB00438

4. General Statistics Office of Vietnam (2020). Monthly average income per capita at current prices by residence and by region by Residence and region and Year. 2020 Jun 08 [Cited 2020 Dec 01]. Available from: https://www.gso.gov.vn/en/pxweb/?pxid=E1127\& theme $=$ Health $\% 2 \mathrm{C} \% 20 \mathrm{C}$ ulture $\% 2 \mathrm{C} \% 20$ Sport $\% 20$ and $\% 20$ Living $\% 20$ st andard

5. Quet F, Vlieghe E, Leyer C, et al. Antibiotic prescription behaviours in Lao People's Democratic Republic: a knowledge, attitude and practice survey. Bull World Health Organ.

2015;93(4):219-227. doi:10.2471/BLT.14.142844

6. The Drugs.com Database. Ceftazidime Dosage. 2020 Sep 01 [Cited 2020 Dec 03]. Available from:

https://www.drugs.com/dosage/ceftazidime.ht ml\#Usual_Pediatric_Dose_for_Pneumonia

7. Vietnam Ministry of Health. Guidelines on antibiotic use. 2015 Jun 19.

8. WHO. Executive summary - The Selection and Use of Essential Medicines 2017, Report of the 21st WHO Expert Committee on the Selection and Use of Essential Medicines, Geneva. 